\title{
Evidence for nevirapine bioactivation in man: Searching for the first step in the mechanism of nevirapine toxicity
}

\author{
Umbelina Caixas $^{\mathrm{a}, \mathrm{b}}$, Alexandra M.M. Antunes ${ }^{\mathrm{c}, *}$, Aline T. Marinho ${ }^{\mathrm{a}}$, Ana L.A. Godinho ${ }^{\mathrm{a}, \mathrm{c}}$, \\ Nádia M. Grilo ${ }^{a}$, M. Matilde Marques ${ }^{c}$, M. Conceição Oliveira ${ }^{c}$, Teresa Branco ${ }^{\mathrm{d}}$, \\ Emília C. Monteiro $^{a}$, Sofia A. Pereira ${ }^{\mathrm{a}, * *}$ \\ a Centro de Estudos de Doenças Crónicas (CEDOC), Faculdade de Ciências Médicas (FCM), Universidade NOVA 1169-056 Lisboa, Portugal \\ ${ }^{\mathrm{b}}$ Centro Hospitalar de Lisboa Central (CHLC), 1150-199 Lisboa, Portugal \\ c Centro de Química Estrutural, Instituto Superior Técnico (CQE-IST), Universidade Técnica de Lisboa, Avenida Rovisco Pais, 1049-001 Lisboa, Portugal \\ d Hospital Prof. Doutor Fernando Fonseca (HFF), 2720-276 Amadora, Portugal
}

\section{A R T I C L E I N F O}

\section{Article history:}

Received 2 May 2012

Received in revised form 19 June 2012

Accepted 20 June 2012

Available online 28 June 2012

\section{Keywords:}

Idiosyncratic reactions

Hepatotoxicity

Bioactivation

Protein adducts

HIV

Nevirapine

\begin{abstract}
A B S T R A C T
Despite its efficacy, including in the prevention of vertical transmission, the antiretroviral nevirapine is associated with severe idiosyncratic hepatotoxicity and skin rash. The mechanisms underlying nevirapine toxicity are not fully understood, but drug bioactivation to reactive metabolites capable of forming stable protein adducts is thought to be involved. This hypothesis is based on the paradigm that drug reactive metabolites have the potential to bind to self-proteins, which results in drug-modified proteins being perceived as foreign by the immune system. The aim of the present work was to identify hemoglobin adducts in HIV patients as biomarkers of nevirapine haptenation upon bioactivation. The ultimate goal is to develop diagnostic methods for predicting the onset of nevirapine-induced toxic reactions.

All included subjects were adults on nevirapine-containing antiretroviral therapy for at least 1 month. The protocol received prior approval from the Hospital Ethics Committees and patients gave their written informed consent. Nevirapine-derived adducts with the $N$-terminal valine of hemoglobin were analyzed by an established liquid chromatography-electrospray ionization-tandem mass spectrometry method and characterized on the basis of retention time and mass spectrometric fragmentation pattern by comparison with adduct standards prepared synthetically. The nevirapine adducts were detected in 12/13 patient samples, and quantified in $11 / 12$ samples ( $2.58 \pm 0.8 \mathrm{fmol} / \mathrm{g}$ of hemoglobin).

This work represents the first evidence of nevirapine-protein adduct formation in man and confirms the ability of nevirapine to modify self-proteins, thus providing clues to the molecular mechanisms underlying nevirapine toxicity. Moreover, the possibility of assessing nevirapine-protein adduct levels has the potential to become useful for predicting the onset of nevirapine-induced adverse reactions.
\end{abstract}

(c) 2012 Published by Elsevier Ireland Ltd.
Abbreviations: cART, combined antiretroviral therapy; CID, collision-induced dissociation; GSH, glutathione; Hb, hemoglobin; HIV, human immunodeficiency virus; HLA, human leukocyte antigen; 12-OH-NVP, 12-hydroxy-nevirapine; LC-ESI-MS/MS, liquid chromatography-electrospray ionization-tandem mass spectrometry; MHC, major histocompatibility complex; NNRTI, non-nucleoside reverse transcriptase inhibitor; NVP, nevirapine.

* Corresponding author. Tel.: +35121 8417627; fax: +351218464455.

** Corresponding author at: Centro de Estudos de Doenças Crónicas (CEDOC), Faculdade de Ciências Médicas (FCM), Universidade NOVA, Campo Mártires da Pátria 130, 1169-056 Lisboa, Portugal. Tel.: +351 21 8803035; fax: +351 218803083.

E-mail addresses: alexandra.antunes@ist.utl.pt (A.M.M. Antunes), sofia.pereira@fcm.unl.pt (S.A. Pereira).

\section{Introduction}

Since 1996, the inception of combined antiretroviral therapy (CART) has changed the prognosis of human immunodeficiency virus (HIV) infection from a lethal disease to a chronic condition in properly medicated patients. However, HIV-positive individuals still face obstacles associated with chronic treatment, including adherence to a daily administration schedule, loss of therapeutic efficacy and drug-induced toxicity. Moreover, increased concerns are emerging, regarding the long-term adverse effects of cART (Powles et al., 2009), that have been linked to the premature onset of aging observed in HIV-infected patients (Deeks, 2009). Powles et al. (2009) showed an epidemiologic association between the chronic treatment with non-nucleoside reverse transcriptase inhibitors (NNRTI) and a higher incidence of non-AIDS-defining cancers. 


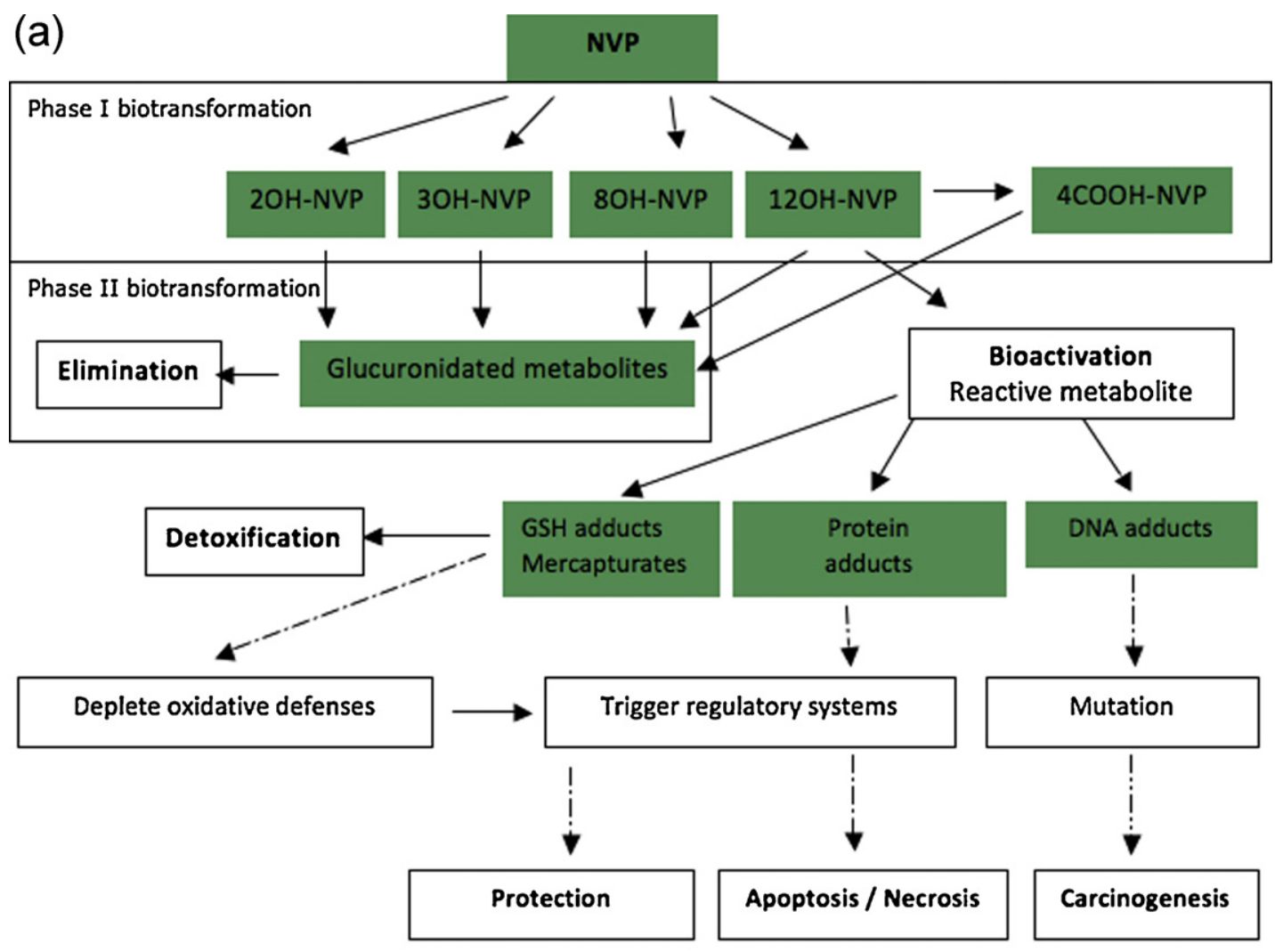

(b)

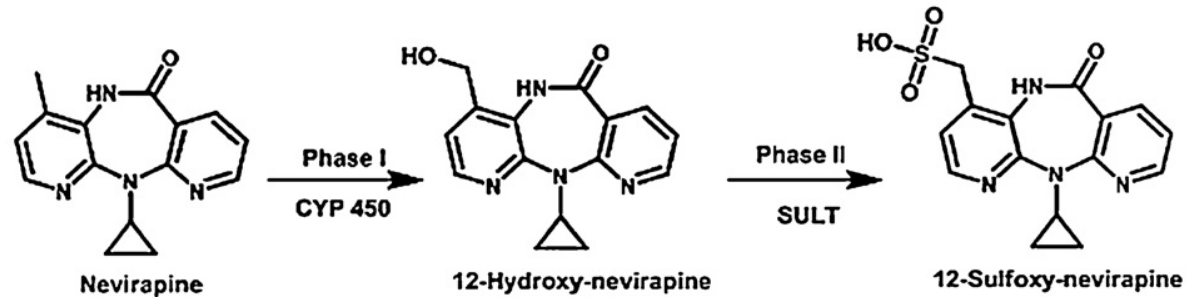

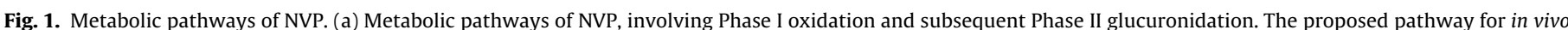

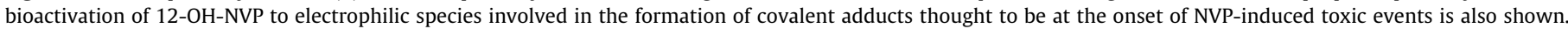

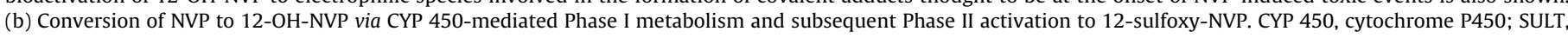
sulfotransferase.

Nevirapine (NVP, Fig. 1) was the first NNRTI approved by the US Food and Drug Administration for the treatment of HIV type-1 infection, as part of cART (Food and Drug Administration, 1996). Currently, NVP is still the most prescribed NNRTI in the world, and remains the most prescribed antiretroviral in countries with limited economic resources, partly due to its low cost (Ades et al., 2000; Lockman et al., 2007). The favorable metabolic profile is one of the therapeutic advantages of NVP (Ruiz et al., 2001; Clotet et al., 2003), rendering it suitable for use in patients with diabetes, dyslipidemia or metabolic syndrome comorbidities. Moreover, the low incidence of adverse drug reactions in the central nervous system (Medrano et al., 2008), allows NVP use in the context of psychiatric disorders or addiction to narcotic drugs. Furthermore, one of the most relevant benefits of NVP is its efficacy in the prevention of motherto-child transmission of the HIV-1 infection, with the drug being commonly prescribed to pregnant women and their children (Ades et al., 2000; Medrano et al., 2008; Perinatal HIV Guidelines Working Group, 2009). However, NVP use has been associated with restrictive idiosyncratic hepatotoxicity and cutaneous hypersensitivity
(Taiwo, 2006; Medrano et al., 2008; De Lazzari et al., 2008). Severe, life-threatening, and even fatal cases of hepatotoxicity (Cattelan et al., 1999) have been described.

Concern about NVP adverse reactions arose following case reports of liver failure in individuals on post-exposure prophylaxis (Johnson and Baraboutis, 2000; Centers for Disease Control and Prevention, 2001) and in asymptomatic HIV-infected patients with well preserved immunity, administered NVP-containing first line cART (Cattelan et al., 1999; Stern et al., 2003). These adverse reactions are more frequent during the first 6 weeks of treatment and women (including those who are pregnant and of Asian ethnicity) seem to be at increased risk of developing NVP-related toxicities (Ho et al., 1998; Antinori et al., 2001; Bersoff-Matcha et al., 2001). Given that immunocompetence is regarded as an additional risk factor for the development of these reactions (De Lazzari et al., 2008), it is recommended that the drug should be initiated in cART-naïve-women with a CD4 cell count below 250 cells $/ \mathrm{mm}^{3}$ and below 400 cells $/ \mathrm{mm}^{3}$ in men (Thompson et al., 2010). For the same reason, NVP is not recommended as part of post-exposure 
prophylaxis (Patel et al., 2004). This association with high CD4 $\mathrm{T}$ cell counts stems from an immune response at the onset of NVP-induced toxicity (Yuan et al., 2011). Although, the specific mechanisms underlying the idiosyncratic toxicity of NVP are still uncertain, several approaches in vitro (Antunes et al., 2008, 2010a,b) and in animal models in vivo (Shenton et al., 2003; Chen et al., 2008) have suggested that bioactivation of the Phase I NVP metabolite, 12-hydroxy-NVP (12-OH-NVP), to reactive electrophiles (e.g., 12-sulfoxy-NVP, Fig. 1) is involved (Antunes et al., 2008, 2010a,b; Chen et al., 2008; Pereira et al., 2012).

Typically, electrophilic drug metabolites are short-lived species in vivo, and thus extremely difficult to detect in humans or experimental animals; however, their propensity to react with bionucleophiles (e.g., amino acids and DNA bases), yielding stable covalent adducts, provides an adequate means for their indirect detection and quantification in body fluids and tissues (Park et al., 2005). Electrophilic metabolites can react with glutathione (GSH), potentially leading to its depletion, and with biomacromolecules (proteins, lipids, and nucleic acids) which can trigger an immune response (Russmann et al., 2009; Park et al., 2011) (Fig. 1). Besides providing clues about the molecular mechanisms underlying drug toxicity, the covalent adducts formed upon reaction with bionucleophiles can be useful markers of toxicity for biomonitoring purposes, especially when well-characterized standards are available (Törnqvist et al., 2002; Angerer et al., 2007; Rubino et al., 2009).

Srivastava et al. (2010) have provided evidence for covalent binding of reactive NVP metabolites to amino acids and peptides (presumably GSH) in vivo, by identifying two NVP mercapturate conjugates in the urine of HIV-positive individuals. However, evidence for NVP metabolism to reactive derivatives with haptenation ability in humans, yielding stable protein adducts, has yet to be provided. Toward this goal, the aim of this work was to investigate whether metabolism to reactive 12-OH-NVP derivatives resulted in detectable adducts with the $N$-terminal valine of hemoglobin (Hb) in HIV-infected patients on NVP-containing cART. Herein is reported the identification of NVP-protein adducts in the blood samples of HIV-infected individuals, thus demonstrating the ability of NVP to modify self-proteins.

\section{Materials and methods}

\subsection{Chemicals and standards}

$\mathrm{NaCl}$ was purchased from Merck KGaA (Darmstadt, Germany) and water was filtered using a Millipore Milli-Q Water Purification System (Billerica, MA). All other reagents were purchased from Sigma-Aldrich Química S.A. (Madrid, Spain) and used as received. The NVP-valine Edman adduct standard was synthesized as described in Antunes et al. (2010a).

\subsection{Study design}

The protocol received prior approval from the Ethics Committees of Centro Hospitalar de Lisboa Central, EPE and Hospital Prof. Doutor Fernando Fonseca, EPE. The patients gave their written informed consent and adherence was controlled through a questionnaire.

All eligible patients ( 9 men and 4 women) were adults with documented HIV-infection who had received continuous treatment with NVP-containing cART regimens ( $200 \mathrm{mg}$ twice daily) for more than 1 month, regardless of past therapeutic history. Exclusion criteria were being under 18 years of age, having AIDS-defining conditions, and compliance issues. The patients' age, sex, ethnicity, and antiretroviral co-medication data were recorded. A control group of 3 healthy volunteers not exposed to NVP was also included in the study.

2.3. Identification and quantification of an NVP-derived adduct with the $\mathrm{N}$-terminal valine of hemoglobin (NVP-valine Edman adduct)

Identification and quantification of the NVP-valine Edman adduct was conducted in three steps: (1) hemoglobin isolation and purification from blood samples; (2) detachment of $\mathrm{N}$-terminal valine adducts from hemoglobin; (3) analysis of the detached adducts by liquid chromatography-electrospray ionization-tandem mass spectrometry (LC-ESI-MS/MS).

\subsubsection{Isolation and purification of hemoglobin}

Blood samples $(2 \mathrm{~mL})$ were collected in heparinized tubes; the samples were centrifuged at $3000 \times g$ for $10 \mathrm{~min}$, to separate plasma from the blood cells. Aliquots of the blood cell layer $(400 \mu \mathrm{L})$ were heated for $60 \mathrm{~min}$ at $60^{\circ} \mathrm{C}$ for HIV inactivation before handling at room temperature. The cell layer was then washed three times with equal volumes of $0.9 \% \mathrm{NaCl}$. The saline solution was discarded and Milli-Q water (1.5 volumes) was added to each sample, to promote cell lysis. A $50 \mathrm{mM} \mathrm{HCl}$ solution in 2-propanol $(6 \mathrm{~mL})$ was subsequently added to $1 \mathrm{~mL}$ of the lysate and the mixture was centrifuged at $3000 \times g$ for $10 \mathrm{~min}$, to remove cell membranes. Ethyl acetate ( 5 volumes) was added then to the supernatant to precipitate $\mathrm{Hb}$ and the pellet was washed with $20 \mathrm{~mL}$ of $n$-pentane and finally dried under reduced pressure.

\subsubsection{Detachment of the $N$-terminal valine from hemoglobin}

After $\mathrm{Hb}$ isolation from the red blood cells each sample was subjected to $\mathrm{N}$-alkyl Edman degradation with phenyl isothiocyanate (Antunes et al., 2010b). Briefly, each $50 \mathrm{mg}$ sample was dissolved in $\mathrm{N}, \mathrm{N}$-dimethylformamide $(1.5 \mathrm{~mL})$, followed by the addition of $1 \mathrm{M} \mathrm{NaOH}(65 \mu \mathrm{L})$ and phenyl isothiocyanate $(10 \mu \mathrm{L})$. The sample was subsequently stirred for $2 \mathrm{~h}$ at $37^{\circ} \mathrm{C}$ and then for $1.5 \mathrm{~h}$ at $45^{\circ} \mathrm{C}$. Upon cooling to room temperature, water $(2 \mathrm{~mL})$ was added, and adducts were extracted with ethyl acetate $(2 \times 1 \mathrm{~mL})$. The organic phase was dried under reduced pressure, and the contents were redissolved in methanol prior to analysis by LC-ESI-MS/MS.

\subsubsection{LC-ESI-MS/MS analyses}

LC-ESI-MS/MS analyses were performed with a ProStar 410 autosampler, two 210-LC chromatography pumps, a ProStar 335 diode array detector, and a 500MS ion trap mass spectrometer, with an ESI ion source (Varian, Inc., Palo Alto, CA). Data acquisition and processing were performed using Varian MS Control 6.9 software. The samples were injected onto the column via a Rheodyne injector with a $20 \mu \mathrm{L}$ loop. Separations were conducted at $30^{\circ} \mathrm{C}$, using a Luna C18 (2) column $(150 \mathrm{~mm} \times 2 \mathrm{~mm}, 3 \mu \mathrm{m}$; Phenomenex, Torrance, $\mathrm{CA})$. The mobile phase was delivered at a flow rate of $200 \mu \mathrm{L} / \mathrm{min}$. Initially, a 5-min isocratic elution with $5 \%$ acetonitrile in $0.1 \%$ aqueous formic acid was conducted, followed by a 30 -min linear gradient from 5 to $70 \%$ acetonitrile, a 2 -min linear gradient to $100 \%$ acetonitrile, and an 8-min isocratic elution with acetonitrile. The mass spectrometer was operated in the positive ESI mode; the optimized operating parameters were ion spray voltage, $+4.8 \mathrm{kV}$; capillary voltage, $10 \mathrm{~V}$; and RF loading, $90 \%$. Nitrogen was used as the nebulizing and drying gas, at pressures of 50 and 30 psi, respectively; the drying gas temperature was $350^{\circ} \mathrm{C}$. MS ${ }^{n}$ spectra were obtained with an isolation window of $1.5 \mathrm{Da}$, excitation energy values between 0.9 and $1.0 \mathrm{~V}$, and an excitation time of $10 \mathrm{~ms}$ (CID up to $\mathrm{MS}^{3}$ ).

The adducts were identified on the basis of retention time and MS fragmentation pattern by comparison with an NVP-valine Edman adduct standard prepared synthetically (Antunes et al., 2010a). Quantification was based upon calibration with this synthetic standard.

\section{Results}

Thirteen HIV-positive patients [nine men and four women; $54 \pm 11$ years old (mean \pm standard deviation)] were included in the present study. Four of the patients were cART-naïve. A tenofovir + emtricitabine combination was the cART-backbone in 7 of the patients; the other cART backbones were equally distributed between lamivudine + zidovudine and lamivudine + abacavir combinations; all regimens included NVP. The patients' demographic and therapeutic data are summarized in Table 1, along with the detected NVP-valine Edman adduct levels (vide infra).

An $N$-alkyl Edman procedure (Antunes et al., 2010b) was used for the specific detachment of NVP adducts with the $N$-terminal valine of $\mathrm{Hb}$ (Fig. 2). Identification of the NVP-valine Edman adducts in the patient samples was based upon undistinguishable mass spectra and identical retention times when compared with the corresponding synthetic standard, prepared and fully characterized as described in Antunes et al. (2010a). Specifically, the samples were subjected to LC-ESI-MS/MS analysis and the MS ${ }^{3}$ transition from the protonated molecule of the NVP-valine Edman adduct $(\mathrm{m} / \mathrm{z}$ 499 ) to the product ion at $m / z 265$, formed by loss of the hydantoin moiety from the protonated molecule, was followed (Fig. 3). Under our chromatographic conditions (cf. Section 2), 12 out of 13 patient samples and the synthetic adduct standard displayed a signal eluting at $27.5 \mathrm{~min}$. For identification purposes, the extracted ion chromatogram of $m / z 237$ was used. Besides the fragment ion at $m / z 265$, the corresponding $\mathrm{MS}^{3}$ spectra consistently presented two other fragment ions, stemming from loss of the cyclopropyl 
Table 1

Patients' demographic and therapeutic data and NVP-derived Edman adduct levels.

\begin{tabular}{|c|c|c|c|c|c|}
\hline Patient & Age (years) & $\operatorname{Sex}^{\mathrm{a}}$ & Ethnicity $^{\mathrm{b}}$ & cART backbone $^{c}$ & NVP-valine Edman adduct (fmol/g Hb) \\
\hline 1 & 74 & $\mathrm{~F}$ & $\mathrm{C}$ & TDF + FTC & 2.41 \\
\hline 2 & 44 & M & $\mathrm{C}$ & $\mathrm{TDF}+\mathrm{FTC}$ & 1.71 \\
\hline 3 & 70 & M & $\mathrm{C}$ & $3 \mathrm{TC}+\mathrm{ABC}$ & $<$ LLOQ $^{\mathrm{e}}$ \\
\hline 4 & 62 & M & $\mathrm{C}$ & $\mathrm{TDF}+\mathrm{FTC}$ & 3.20 \\
\hline 5 & 54 & M & C & $3 \mathrm{TC}+\mathrm{ABC}$ & 1.59 \\
\hline 6 & 63 & $\mathrm{~F}$ & $\mathrm{C}$ & $3 \mathrm{TC}+\mathrm{AZT}$ & $N D^{f}$ \\
\hline 7 & 54 & M & $\mathrm{C}$ & $3 \mathrm{TC}+\mathrm{AZT}$ & 2.64 \\
\hline 8 & 41 & M & $\mathrm{C}$ & $\mathrm{TDF}+\mathrm{FTC}$ & 4.61 \\
\hline 9 & 45 & $\mathrm{M}$ & $\mathrm{C}$ & $3 \mathrm{TC}+\mathrm{AZT}$ & 2.45 \\
\hline 10 & 52 & M & A & $\mathrm{TDF}+\mathrm{FTC}$ & 2.26 \\
\hline 11 & 43 & $\mathrm{~F}$ & $\mathrm{C}$ & $\mathrm{TDF}+\mathrm{FTC}$ & 2.18 \\
\hline 12 & 40 & M & A & $\mathrm{TDF}+\mathrm{FTC}$ & 2.33 \\
\hline 13 & 61 & $\mathrm{~F}$ & $\mathrm{C}$ & $3 \mathrm{TC}+\mathrm{ABC}$ & 3.03 \\
\hline
\end{tabular}

a $\mathrm{F}$, female; $\mathrm{M}$, male.

b C, Caucasian; A, African.

c TDF, tenofovir; FTC, emtricitabine; 3TC, lamivudine; AZT, zidovudine; ABC, abacavir.

d $\mathrm{Hb}$ was precipitated and subjected to $\mathrm{N}$-alkyl-Edman degradation. The NVP-Edman adducts were quantified as described in Section 2.

e LLOQ lower limit of quantification.

f ND, not detected.

moiety $(m / z 223)$ and loss of carbon monoxide $(m / z 237)$ from the product ion with $m / z 265$ (Fig. 3). These signals were absent from all the control samples. These observations are illustrated in Fig. 4. The extracted ion chromatogram $(\mathrm{m} / z$ 237) and MS/MS spectra resulting from analysis of samples from two patients and a healthy volunteer are presented in panels a, b and c, respectively. The chromatogram and MS/MS spectrum resulting from the synthetic Edman adduct standard are displayed in panel d. As indicated above, NVP-derived Edman adducts with the $\mathrm{N}$-terminal valine of $\mathrm{Hb}$ were identified in all but one patient (a 63-year-old Caucasian woman, on a 3TC + AZT cART regimen). Moreover, based upon calibration with the synthetic standard, the adduct levels could be estimated in samples from 11 patients (Table 1). Despite some variability between patients, which could not be correlated with individual characteristics or therapies due to the small sample size, the results showed a mean adduct level of $2.58 \pm 0.8 \mathrm{fmol} / \mathrm{g}$ of $\mathrm{Hb}$.

\section{Discussion}

Increasing evidence shows that allergic idiosyncratic NVP reactions, characterized by the presence of typical symptoms and signs of adaptive immune responses, including hepatotoxicity, fever, skin reactions and eosinophilia, are genetically determined. It seems that NVP-specific antigens may trigger an immunological response, as shown by screening of human leukocyte antigen (HLA) markers [located within the class I and class II regions of the major histocompatibility complex (MHC)] (Gangar et al., 2000; Pirmohamed and Park, 2001; Mallal et al., 2002; Martin et al., 2005). Recently, a hapten/prohapten hypothesis was proposed for another antiretroviral drug, abacavir, that is also associated with hypersensitivity syndrome (Bharadwaj et al., 2012). According to this model, biotransformation of the drug yields a reactive metabolite, which in turn modifies a cellular protein. This modified protein might undergo proteasome-mediated degradation and produce small peptide fragments, including the haptenated peptide. This peptide will be then recognized by a specific HLA allotype. The time dependence of this process is consistent with a delay between drug administration and the presence of the immunogenic ligand at the cell surface.

An immune role has indeed been proposed for the toxic outcomes of NVP (Yuan et al., 2011), with higher CD4 ${ }^{+}$cell counts being associated with the onset of hepatotoxic reactions (De Lazzari et al., 2008). These observations are suggestive of immune tolerance in patients with severe immunodeficiency. The adverse reactions are, in turn, more frequent and severe in immunologically uncompromised individuals on NVP as prophylaxis for HIV infection (Bharadwaj et al., 2012). Furthermore, NVP-related hepatotoxicity seems to be linked to MHC class II, which presents antigens to $\mathrm{CD}^{+}{ }^{+}$T cells (Yuan et al., 2011); also, both MCH class I and class II polymorphisms have been linked with NVP-related adverse reactions (Martin et al., 2005; Chantarangsu et al., 2009). These reports suggest that NVP-modified self-peptides, presented by MHC class I or class II molecules, are recognized by T cells in the context of particular MHC products. NVP-related rash and hepatitis have been associated with various HLA alleles, which seem to be different in Australian (Martin et al., 2005), Japanese (Gatanaga et al., 2007), Sardinian (Littera et al., 2006), Thai (Chantarangsu et al., 2009; Likanonsakul et al., 2009), and French (Vitezica et al., 2008) populations. Thus, the existence of a different immunogenetic basis for NVP toxicity in different populations cannot be excluded. Likewise, NVP bioactivation, namely via CYP450-mediated formation of reactive metabolites, can vary among ethnic groups (Stöhr et al., 2008; Wyen et al., 2008; Brown et al., 2012).

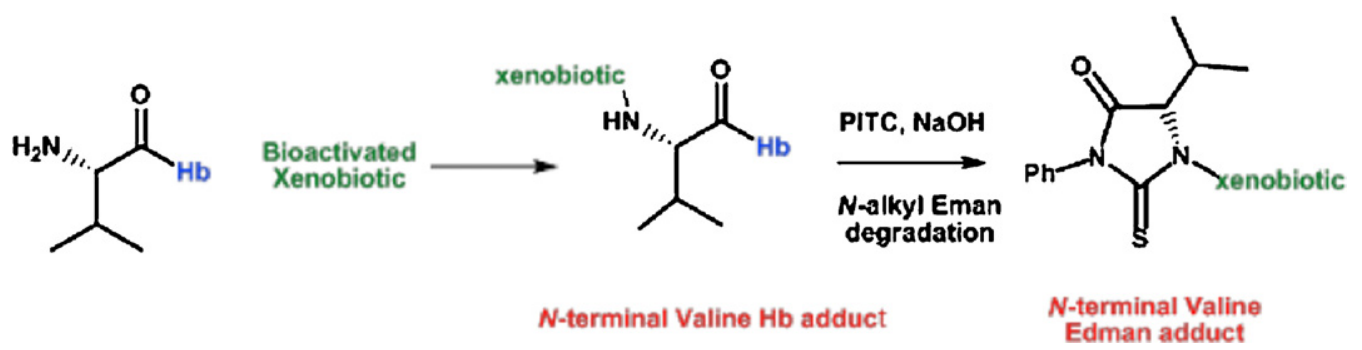

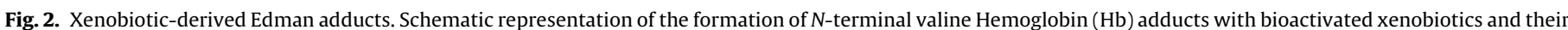
detachment from the protein upon $N$-alkyl Edman degradation. PTIC, phenyl isothiocyanate. 


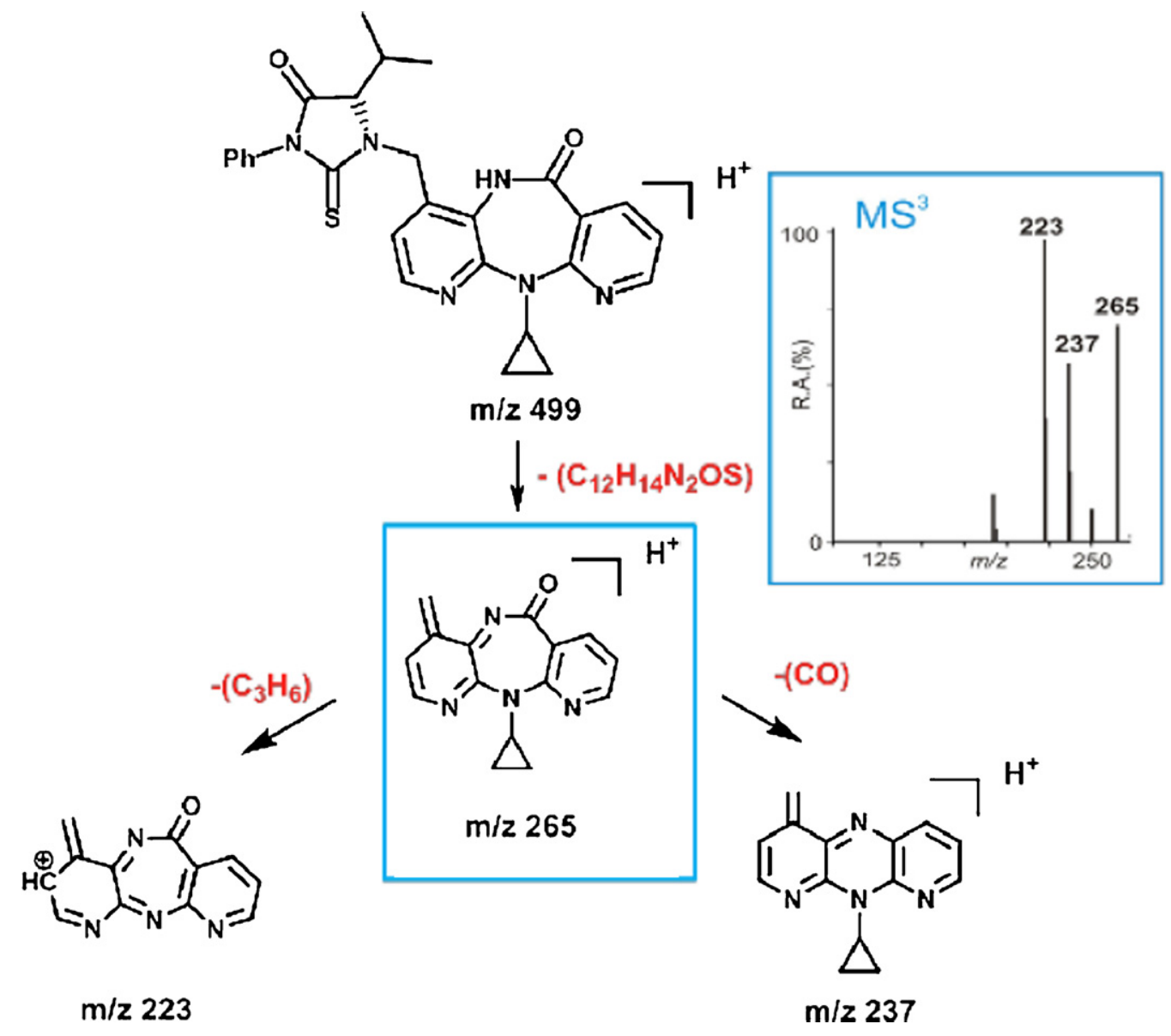

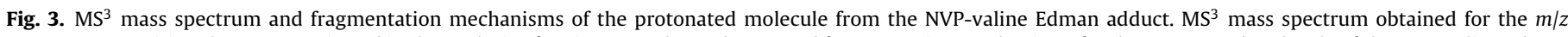

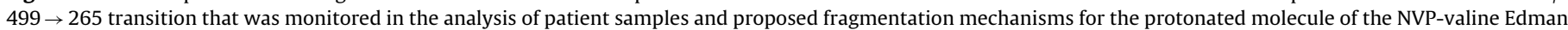
adduct $(m / z 499)$.

In view of the evidence, it is reasonable to assume that NVPinduced adverse reactions will depend upon the ability of reactive NVP metabolites to interact with self-proteins involved in the immune response, yielding covalent adducts. Unlike the parent drugs or their metabolites, covalent adducts (haptens) are widely recognized to interact directly with immune receptors, generating antigens (Park et al., 2011). Nonetheless, the question of how the NVP-specific antigen is generated and the reason(s) why its presentation is restricted to specific HLA allotypes has yet to be clarified. To address this issue it is necessary to start by identifying adducts from reactive NVP metabolites in patients on NVP, as first evidence that this antiretroviral drug is activated in vivo to derivatives with the potential to form an immunogenic drug-peptide complex.

The present manuscript provides the first unequivocal evidence for the ability of reactive metabolites from the Phase I NVP product, 12-OH-NVP, to form haptens (covalent adducts) with proteins in humans. Although we used a model protein (hemoglobin, $c f$. below), a similar mechanism can conceivably be at play with toxicologically relevant proteins, leading to haptens with immunogenic potential. The current work took advantage of the availability, within our research team, of a synthetic adduct standard prepared through reaction of 12-mesyloxy-NVP (a synthetic surrogate for the Phase II metabolite, 12-sulfoxy-NVP) with ethyl valinate. Following $\mathrm{N}$-alkyl Edman degradation, the NVP-valine Edman adduct standard was isolated and fully characterized by mass spectrometry and nuclear magnetic resonance (Antunes et al., 2010a). The availability of this adduct standard allowed the development of a suitable mass spectrometry-based analytical tool, used in the current work to assess this potential biomarker of NVP toxicity in vivo.

NVP-valine Edman adducts were detected in 12 (and quantified in 11) out of $13 \mathrm{Hb}$ samples from HIV-positive patients currently on NVP-containing cART regimens (Table 1). Moreover, none of the 3 healthy volunteers unexposed to NVP had detectable levels of the NVP-specific adduct. The consistent detection of this adduct in the patient samples provides unambiguous proof of NVP bioactivation via the 12-OH-NVP pathway and may be a relevant clue to the molecular mechanisms underlying NVP allergic reactions.

As indicated above, we used $\mathrm{Hb}$ as a model protein. Covalent adducts formed with abundant and easily accessible blood proteins, like $\mathrm{Hb}$ and human serum albumin, can be good biomarkers of drug bioactivation to reactive metabolites and drug proteinmodification and are frequently used to study the ability of drug metabolites to undergo protein haptenation. In particular, adducts formed by $\mathrm{N}$-terminal valine residues of $\mathrm{Hb}$ have been extensively applied for that purpose (Boysen et al., 2007; Chevolleau et al., 2007). The widespread use of these species can be explained on the basis of the high accessibility of xenobiotics to $N$-terminal valine residues in $\mathrm{Hb}$ and the fact that a large fraction of these residues are unionized, and therefore nucleophilic, at blood $\mathrm{pH}$ (Moll and Elfarra, 1999; Törnqvist et al., 2002), which explains why they are especially prone to react with electrophilic metabolites forming covalent adducts. Moreover, the availability of the $\mathrm{N}$-alkyl Edman method (Fig. 2), a mild, simple, wide-ranging, and sensitive post-modification procedure, that allows the selective detachment 


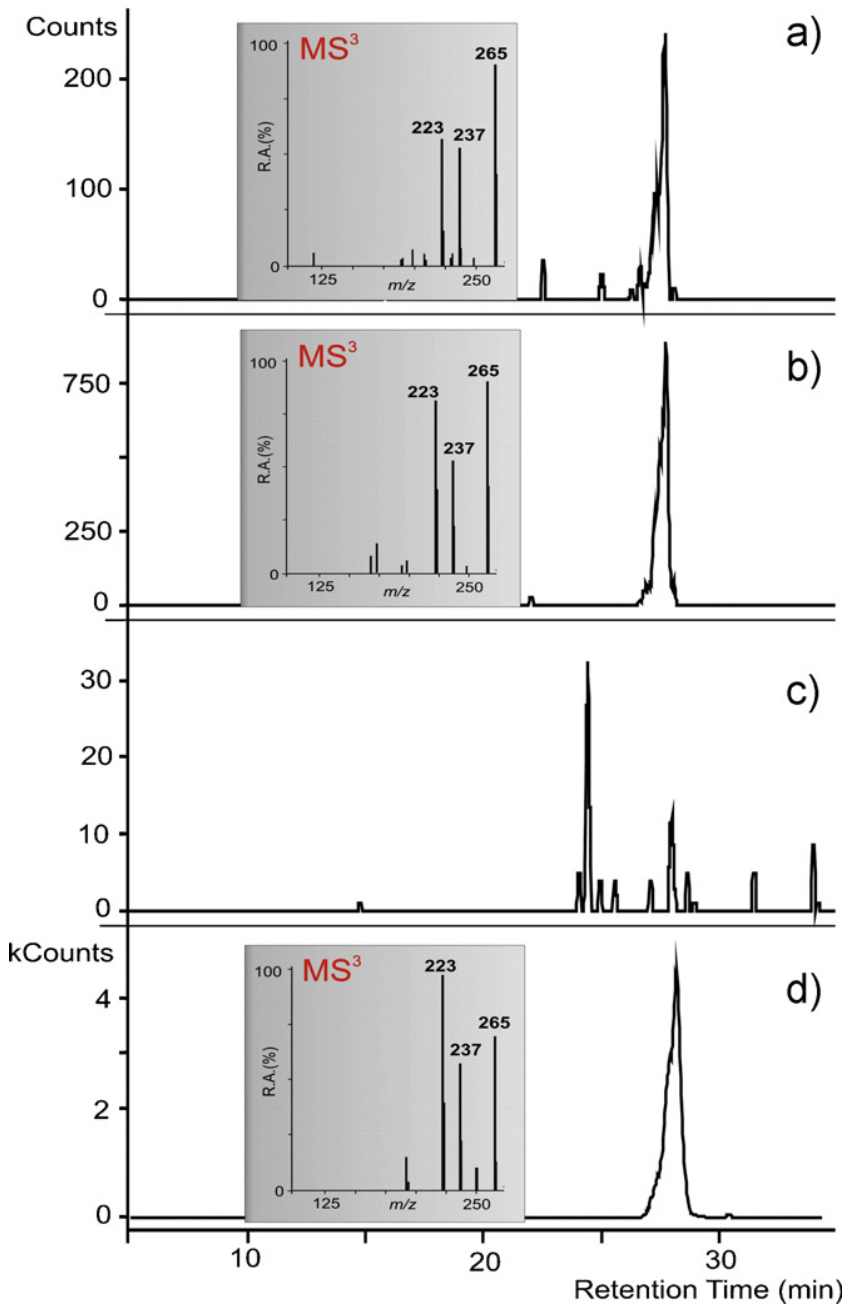

Fig. 4. Representative LC-ESI-MS/MS ion chromatograms obtained upon analysis of the samples. Extracted ion chromatograms ( $m / z$ 237) obtained upon LC-ESI-MS/MS analysis of $\mathrm{m} / \mathrm{z} 265$ and MS/MS mass spectra of: (a) patient 3; (b) patient 9; (c) healthy volunteer unexposed to NVP; (d) synthetic standard. The elution conditions are outlined in Section 2.

of the $N$-terminal valine adducts (as hydantoins, i.e., $N$-terminal valine Edman adducts) from the protein enables adduct detection by mass spectrometry-based methods with a high degree of sensitivity (Törnqvist et al., 2002; Chevolleau et al., 2007). Moreover, $\mathrm{Hb}$ is a good model for studying exposure to electrophiles with potential to induce DNA damage, since the reactive species have to cross the cell membrane to reach $\mathrm{Hb}$. Therefore, if a reactive intermediate forms covalent adducts with $\mathrm{Hb}$ it may eventually reach DNA (Skipper et al., 1994; Kensler et al., 1996; Wild and Pisani, 1997) and form DNA adducts, that could ultimately be involved in tumor initiation. It is worth mentioning that we have demonstrated the ability of 12-OH-NVP derivatives to form stable adducts with deoxynucleosides and DNA (Antunes et al., 2008).

Although NVP mercapturates have previously been identified by Srivastava et al. (2010) in the urine of HIV-positive individuals, it should be noted that these species may not mirror accurately the full extent of NVP bioactivation. Indeed, HIV-infected individuals have depleted GSH levels (Townsend et al., 2003). This depletion may prevent an efficient detoxification of reactive metabolites, which will be more available to react with other biomolecules (e.g. proteins); consequently, a higher risk of hepatotoxicity in these patients is conceivable. As such, in addition to being good models to study protein haptenation by reactive metabolites, blood protein adducts are anticipated to reflect the extent of NVP bioactivation more adequately than urinary mercapturates. Additional assets are the simplicity of sample treatment to assess $\mathrm{N}$-terminal valine adducts from red blood cells and the advantage of selectively extracting these adducts to an organic solvent, which minimizes matrix interferences and enables high levels of sensitivity by mass spectrometry-based analytical methods; by contrast, the analysis of urinary mercapturates frequently deals with matrix interferences. Taken together, these considerations favor the use of $\mathrm{N}$-terminal valine $\mathrm{Hb}$ adducts, over that of mercapturate conjugates, as accurate biomarkers of NVP bioactivation/toxicity.

In summary, using $\mathrm{Hb}$ as a surrogate for toxicologically relevant proteins, we provide herein the first unequivocal evidence for NVP bioactivation to protein-binding metabolites in humans. Further studies with larger cohorts are needed. A more extensive data set should provide a better insight into the mechanisms underlying NVP toxicity, ultimately allowing the development of a screening test to help prevent NVP-associated hypersensitive reactions, including severe liver injury. Clearly, much work is still warranted before progress in tailoring NVP treatment to a reliable risk/benefit balance in individual patients can be achieved.

\section{Conclusion}

The present manuscript provides the first evidence for the ability of 12-OH-NVP-derived reactive metabolites to form adducts with proteins in humans. The consistent detection of this adduct in the patient samples provides unequivocal proof of NVP bioactivation, via the 12-OH-NVP pathway, and may be a relevant clue to the molecular mechanisms underlying NVP-related adverse reactions. This approach can lead to a better understanding of the long-term risks of NVP bioactivation in chronic therapies and ultimately allow the development of screening tests to help prevent idiosyncratic NVP reactions, including severe hepatic damage.

\section{Role of the funding source}

This work was supported by Fundação para a Ciência e a Tecnologia (FCT, Portugal), through research grants (PTDC/SAU-TOX/111663/2009 and PTDC/QUI-QUI/113910/2009) and pluriannual funds to Centro de Química Estrutural (PEstOE/QUI/UI0100/2011).

\section{Conflict of interest statement}

The authors declare that there are no conflicts of interest.

\section{Acknowledgements}

Thanks are due to the Portuguese NMR and MS Networks (ISTUTL Center) for providing access to the facilities and to Inês Faustino (FCM) for technical support.

\section{References}

Ades, A.E., Ratcliffe, J., Gibb, D.M., Sculpher, M.J., 2000. Economic issues in the prevention of vertical transmission of HIV. Pharmacoeconomics 18, 9-22.

Angerer, J., Ewers, U., Wilhelm, M., 2007. Human biomonitoring: state of the art. Int J. Hyg. Environ. Health 210, 201-228.

Antinori, A., Baldini, F., Girardi, E., et al., 2001. Female sex and the use of anti-allergic agents increase the risk of developing cutaneous rash associated with nevirapine therapy. AIDS 15, 1579-1581.

Antunes, A.M.M., Duarte, M.P., Santos, P.P., et al., 2008. Synthesis and characterization of DNA adducts from the HIV reverse transcriptase inhibitor nevirapine. Chem. Res. Toxicol. 21, 1443-1456.

Antunes, A.M.M., Godinho, A.L.A., Martins, I.L., et al., 2010a. Amino acid adduct formation by the nevirapine metabolite, 12-hydroxynevirapine-a possible factor in nevirapine toxicity. Chem. Res. Toxicol. 23, 888-899. 
Antunes, A.M.M., Godinho, A.L.A., Martins, I.L., et al., 2010b. Protein adducts as prospective biomarkers of nevirapine toxicity. Chem. Res. Toxicol. 23, 1714-1725.

Bersoff-Matcha, S.J., Miller, W.C., Aberg, J.A., et al., 2001. Sex differences in nevirapine rash. Clin. Infect. Dis. 32, 124-129.

Bharadwaj, M., Illing, P., Theodossis, A., et al., 2012. Drug hypersensitivity and human leukocyte antigens of the major histocompatibility complex. Annu. Rev. Pharmacol. Toxicol. 52, 401-431.

Boysen, G., Georgieva, N.I., Upton, P.B., et al., 2007. N-terminal globin adducts as biomarkers for formation of butadiene derived epoxides. Chem. Biol. Interact. 166, 84-92.

Brown, K.C., Hosseinipour, M.C., Hoskins, J.M., et al., 2012. Exploration of CYP450 and drug transporter genotypes and correlations with nevirapine exposure in Malawians. Pharmacogenomics 13, 113-121.

Cattelan, A.M., Erne, E., Salatino, A., et al., 1999. Severe hepatic failure related to nevirapine treatment. Clin. Infect. Dis. 29, 455-456.

Centers for Disease Control and Prevention (CDC), 2001. Serious adverse events attributed to nevirapine regimens for postexposure prophylaxis after HIV exposures-worldwide, 1997-2000. Morb. Mortal. Wkly. Rep. 49, 1153-1156.

Chantarangsu, S., Mushiroda, T., Mahasirimongkol, S., et al., 2009. HLA-B*3505 allele is a strong predictor for nevirapine-induced skin adverse drug reactions in HIVinfected Thai patients. Pharmacogenet. Genomics 19, 139-146.

Chen, J., Mannargudi, B.M., Xu, L., Uetrecht, J., 2008. Demonstration of the metabolic pathway responsible for nevirapine-induced skin rash. Chem. Res. Toxicol. 21, 1862-1870.

Chevolleau, S., Jacques, C., Canlet, C., et al., 2007. Analysis of hemoglobin adducts of acrylamide and glycidamide by liquid chromatography-electrospray ionization tandem mass spectrometry, as exposure biomarkers in French population. J. Chromatogr. A 1167, 125-134.

Clotet, B., van der Valk, M., Negredo, E., Reiss, P., 2003. Impact of nevirapine on lipid metabolism. J. Acquir. Immune Defic. Syndr. 34 (Suppl. 1), S79-S84.

De Lazzari, E., León, A., Arnaiz, J.A., et al., 2008. Hepatotoxicity of nevirapine in virologically suppressed patients according to gender and CD4 cell counts. HIV Med. 9, 221-226.

Deeks, S.G., 2009. Immune dysfunction, inflammation, and accelerated aging in patients on antiretroviral therapy. Top. HIV Med. 17, 118-123.

Food and Drug Administration, 1996. FDA approves first new class of HIV drugs. AIDS Alert 11, 89

Gangar, M., Arias, G., O’Brien, J.G., Kemper, C.A., 2000. Frequency of cutaneous reactions on rechallenge with nevirapine and delavirdine. Ann. Pharmacother. 34 839-842.

Gatanaga, H., Yazaki, H., Tanuma, J., et al., 2007. HLA-Cw8 primarily associated with hypersensitivity to nevirapine. AIDS 21, 264-265.

Ho, T.T., Wong, K.H., Chan, K.C., Lee, S.S., 1998. High incidence of nevirapineassociated rash in HIV-infected Chinese. AIDS 12, 2082-2083.

Johnson, S., Baraboutis, J.G., 2000. Adverse effects associated with use of nevirapine in HIV postexposure prophylaxis for 2 health care workers. JAMA 284, 2722-2723.

Kensler, T.W., Groopman, J.D., Wogan, G.N., 1996. Use of carcinogen-DNA and carcinogen-protein adduct biomarkers for cohort selection and as modifiable end points in chemoprevention trials. IARC Sci. Publ. 139, 237-248.

Likanonsakul, S., Rattanatham, T., Feangvad, S., et al., 2009. HLA-Cw*04 allele associated with nevirapine-induced rash in HIV-infected Thai patients. AIDS Res. Ther. 6,22

Littera, R., Carcassi, C., Masala, A., et al., 2006. HLA-dependent hypersensitivity to nevirapine in Sardinian HIV patients. AIDS 20, 1621-1626.

Lockman, S., Shapiro, R.L., Smeaton, L.M., et al., 2007. Response to antiretroviral therapy after a single, peripartum dose of nevirapine. N. Engl. J. Med. 356, 135-147.

Mallal, S., Nolan, D., Witt, C., et al., 2002. Association between presence of HLA-B*5701, HLA-DR7, and HLA-DQ3 and hypersensitivity to HIV-1 reversetranscriptase inhibitor abacavir. Lancet 359 (9308), 727-732.

Martin, A.M., Nolan, D., James, I., et al., 2005. Predisposition to nevirapine hypersensitivity associated with HLA-DRB1*0101 and abrogated by low CD4 T-cell counts. AIDS 19, 97-99.

Medrano, J., Barreiro, P., Tuma, P., et al., 2008. Risk for immune-mediated liver reactions by nevirapine revisited. AIDS Rev. 10,110-115.

Moll, T.S., Elfarra, A.A. 1999. Characterization of the reactivity, regioselectivity, and stereoselectivity of the reactions of butadiene monoxide with valinamide and the N-terminal valine of mouse and rat hemoglobin. Chem. Res. Toxicol. 12 679-689.

Park, B.K., Kitteringham, N.R., Maggs, J.L., et al., 2005. The role of metabolic activation in drug-induced hepatotoxicity. Annu. Rev. Pharmacol. Toxicol. 45, 177-202.

Park, B.K., Laverty, H., Srivastava, A., et al., 2011. Drug bioactivation and protein adduct formation in the pathogenesis of drug-induced toxicity. Chem. Biol. Interact. $192,30-36$

Patel, S.M., Johnson, S., Belknap, S.M., et al., 2004. Serious adverse cutaneous and hepatic toxicities associated with nevirapine use by non-HIV-infected individuals. J. Acquir. Immune Defic. Syndr. 35, 120-125.

Pereira, S.A., Marques, M.M., Caixas, U., et al., 2012. Understanding the molecular basis for the hazards associated with nevirapine treatment. In: Berhardt, L.V (Ed.), Advances in Medicine and Biology, vol. 44. Nova Science Publishers, Inc. Hauppauge, NY, ISBN 978-1-62100-961-0.

Perinatal HIV Guidelines Working Group, 2009. Public Health Service Task Force Recommendations for Use of Antiretroviral Drugs in Pregnant HIV-infected Women for Maternal Health and Interventions to Reduce Perinatal HIV Transmission in the United States, pp. 1-90. Available at: http://aidsinfo.nih.gov/ContentFiles/ PerinatalGL.pdf (accessed 01.19.2012).

Pirmohamed, M., Park, B.K., 2001. HIV and drug allergy. Curr. Opin. Allergy Clin. Immunol. 1, 311-316.

Powles, T., Robinson, D., Stebbing, J., et al., 2009. Highly active antiretroviral therapy and the incidence of non-AIDS-defining cancers in people with HIV infection. J Clin. Oncol. 27, 884-890.

Rubino, F.M., Pitton, M., Di Fabio, D., Colombi, A., 2009. Toward an "omic" physiopathology of reactive chemicals: thirty years of mass spectrometric study of the protein adducts with endogenous and xenobiotic compounds. Mass Spectrom. Rev. 28, 725-784.

Ruiz, L., Negredo, E., Domingo, P., et al., 2001. Antiretroviral treatment simplification with nevirapine in protease inhibitor-experienced patients with HIV-associated lipodystrophy: 1-year prospective follow-up of a multicenter, randomized, controlled study. J. Acquir. Immune Defic. Syndr. 27, 229-236.

Russmann, S., Kullak-Ublick, G.A., Grattagliano, I., 2009. Current concepts of mechanisms in drug-induced hepatotoxicity. Curr. Med. Chem. 16, 3041-3053.

Shenton, J.M., Teranishi, M., Abu-Asab, M.S., et al., 2003. Characterization of a potential animal model of an idiosyncratic drug reaction: nevirapine-induced skin rash in the rat. Chem. Res. Toxicol. 16, 1078-1089.

Skipper, P.L., Peng, X., Soohoo, C.K., Tannenbaum, S.R., 1994. Protein adducts as biomarkers of human carcinogen exposure. Drug Metab. Rev. 26, 111-124.

Srivastava, A., Lian, L.Y., Maggs, J.L., et al., 2010. Quantifying the metabolic activation of nevirapine in patients by integrated applications of NMR and mass spectrometries. Drug Metab. Dispos. 38, 122-132.

Stern, J.O., Robinson, P.A., Love, J., et al., 2003. A comprehensive hepatic safety analysis of nevirapine in different populations of HIV infected patients. J. Acquir. Immune Defic. Syndr. 34 (Suppl 1), 21-33.

Stöhr, W., Back, D., Dunn, D., et al., 2008. Factors influencing efavirenz and nevirapine plasma concentration: effect of ethnicity, weight and co-medication. Antivir. Ther. 13, 675-685

Taiwo, B.O., 2006. Nevirapine toxicity. Int. J. STD AIDS 17, 364-370.

Thompson, M.A., Aberg, J.A., Cahn, P., et al., 2010. Antiretroviral treatment of adult HIV infection: 2010 recommendations of the International AIDS Society-USA panel. JAMA 304, 321-333.

Törnqvist, M., Fred, C., Haglund, J., et al., 2002. Protein adducts: quantitative and qualitative aspects of their formation, analysis and application. J. Chromatogr. B 778, 279-308

Townsend, D.M., Tew, K.D., Tapiero, H., 2003. The importance of glutathione in human disease. Biomed. Pharmacother. 57, 145-155.

Vitezica, Z.G., Milpied, B., Lonjou, C., et al., 2008. HLA-DRB1*01 associated with cutaneous hypersensitivity induced by nevirapine and efavirenz. AIDS 22 540-541.

Wild, C.P., Pisani, P., 1997. Carcinogen-DNA and carcinogen-protein adducts in molecular epidemiology. IARC Sci. Publ. 142, 143-158.

Wyen, C., Hendra, H., Vogel, M., et al., 2008. Impact of CYP2B6 983T>C polymorphism on non-nucleoside reverse transcriptase inhibitor plasma concentrations in HIVinfected patients. J. Antimicrob. Chemother. 61, 914-918.

Yuan, J., Guo, S., Hall, D., et al., 2011. Toxicogenomics of nevirapine-associated cutaneous and hepatic adverse events among populations of African, Asian, and European descent. AIDS 25, 1271-1280. 